SUBJECT AREAS:

MATERIALS FOR OPTICS

BIOTECHNOLOGY

OPTICAL MANIPULATION AND

TWEEZERS

APPLIED PHYSICS

SCIENTIFIC REPORTS:

$3: 1258$

DOI: $10.1038 /$ srep0 1258

(2013)

Published: 13 February 2013

Updated:

1 March 2013

Correspondence and requests for materials should be addressed to C.L. (carlo.liberale@iit.

it)

* Current address: Max Planck Institute of Molecular Cell Biology and Genetics MPICBG, Pfotenhauer str. 108, Dresden 01307 ,

Germany.

† Current address: Istituto di Fotonica e Nanotecnologie (IFN) -CNR, Dipartimento di Fisica, Politecnico di Milano, P.zza Leonardo da Vinci 32, 20133 Milano, Italy.

\section{ERRATUM: Integrated microfluidic device for single-cell trapping and spectroscopy}

\author{
C. Liberale' , G. Cojoc ${ }^{2 *}$, F. Bragheri $3 \$$, P. Minzioni ${ }^{3}$, G. Perozziello² ${ }^{2}$ R. La Rocca' ${ }^{1}$, L. Ferrara ${ }^{1,3}$, \\ V. Rajamanickam', E. Di Fabrizio ${ }^{2,4} \&$ I. Cristiani ${ }^{3}$
}

${ }^{1}$ Nanostructures, Istituto Italiano di Tecnologia, Via Morego 30, 16163 Genova, Italy, ${ }^{2}$ BioNEM Lab, Dipartimento di Medicina Sperimentale e Clinica, Università Magna Graecia di Catanzaro, 88100 Catanzaro, Italy, ${ }^{3}$ Dipartimento di Ingegneria Industriale e dell'Informazione, Università di Pavia, Via Ferrata 1, 27100 Pavia, Italy, ${ }^{4}$ KAUST (King Abdullah University of Science and Technology), PSE and BESE divisions, Jeddah, Saudi Arabia.

Due to a typesetting error, the authors C. Liberale, R. La Rocca, L. Ferrara and V. Rajamanickam were incorrectly listed as being affiliated with KAUST (King Abdullah University of Science and Technology), PSE and BESE divisions, Jeddah, Saudi Arabia. This has now been corrected in both the PDF and HTML versions of the Article. 